\title{
Two Cases of Granulomatous Mastitis Caused by Corynebacterium kroppenstedtii Infection in Nulliparous Young Women with Hyperprolactinemia
}

\author{
Satoshi Kutsuna ${ }^{1}$, Kazuhisa Mezaki ${ }^{2}$, Maki Nagamatsu ${ }^{1,3}$, Junwa Kunimatsu ${ }^{4}$, \\ Kei Yamamoto ${ }^{1}$, Yoshihiro Fujiya ${ }^{1}$, Momoko Mawatari ${ }^{1}$, Nozomi Takeshita ${ }^{1}$, \\ Kayoko Hayakawa ${ }^{1}$, Yasuyuki Kato ${ }^{1}$, Shuzo Kanagawa ${ }^{1}$ and Norio Ohmagari ${ }^{1}$
}

\begin{abstract}
Recently, an association between granulomatous mastitis and local infection with Corynebacterium $(C$. $)$ kroppenstedtii has been suggested. We herein report two cases of granulomatous mastitis resulting from $C$. kroppenstedtii infection in nulliparous young women with hyperprolactinemia. Both cases involved nulliparous patients with drug-induced hyperprolactinemia, and both individuals received incision and drainage, after which the pus was sent to our laboratory. Corynebacterium spp. grew on blood agar, and 16S rRNA gene sequencing identified the pathogen as C. kroppenstedtii. In conclusion, lactational changes caused by druginduced hyperprolactinemia may increase the risk of granulomatous mastitis after $C$. kroppenstedtii infection.
\end{abstract}

Key words: granulomatous mastitis, Corynebacterium kroppenstedtii, 16S rRNA gene sequencing, hyperprolactinemia

(Intern Med 54: 1815-1818, 2015)

(DOI: 10.2169/internalmedicine.54.4254)

\section{Introduction}

Granulomatous mastitis, an inflammatory disease of unknown etiology, is an uncommon benign disorder of the breast (1). Recently, an association of this condition with local infection with Corynebacterium $(C$.) kroppenstedtii has been suggested. In 2002, Paviour et al. reported 24 patients with mastitis (2), among whom Corynebacterium spp. were isolated from breast tissue, pus and/or deep wound swabs, with $C$. kroppenstedtii being the most commonly identified pathogen. In this report, we describe two cases of granulomatous mastitis due to $C$. kroppenstedtii infection identified on $16 \mathrm{~S}$ rRNA gene sequencing.

\section{Case Reports}

\section{Case 1}

A 20-year-old nulliparous Japanese woman presented to our hospital with recurrent mastitis in October 2013. Prior to this consultation, she had experienced two episodes of mastitis in March and October 2012, after which she was diagnosed with granulomatous mastitis and treated with ultrasound-guided aspiration drainage. She had also been diagnosed with mixed connective tissue disease at 15 years of age and had been taking prednisolone ( $5 \mathrm{mg} /$ day), azathioprine $(50 \mathrm{mg} /$ day), alfacalcidol and sulpiride since that time. She was not allergic to any medications and did not smoke or drink alcohol. She was nulliparous and not pregnant. The findings of a physical examination were normal, except for

\footnotetext{
${ }^{1}$ Disease Control and Prevention Center, National Center for Global Health and Medicine, Japan, ${ }^{2}$ Microbiology Laboratory, National Center for Global Health and Medicine, Japan, ${ }^{3}$ Department of Infectious Diseases, Research Institute, National Center for Global Health and Medicine, Japan and ${ }^{4}$ Department of General Medicine, National Center for Global Health and Medicine, Japan Received for publication October 9, 2014; Accepted for publication November 24, 2014 Correspondence to Dr. Satoshi Kutsuna, sonare.since1192@gmail.com
} 


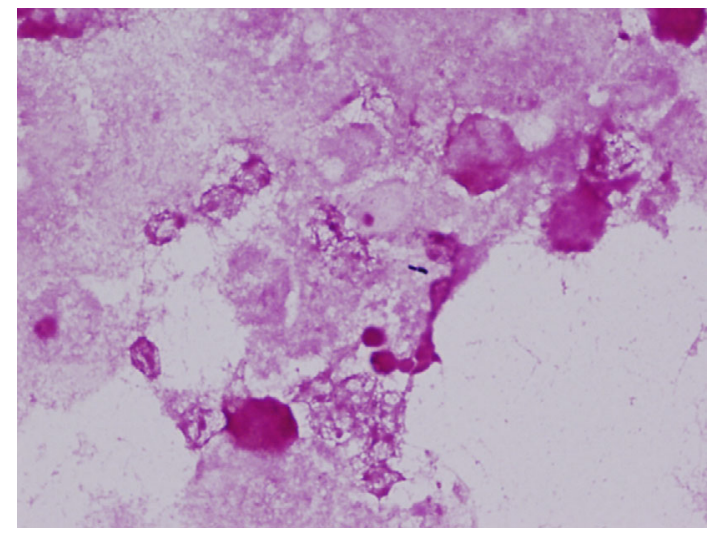

Figure 1. Gram staining of the pus with a number of neutrophils and a few Gram-positive bacilli (Case 1).

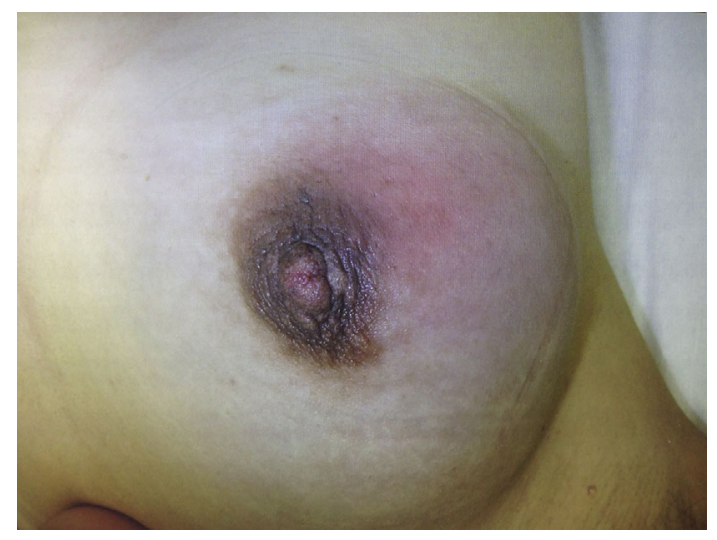

Figure 2. Right breast of the patient exhibiting redness and swelling (Case 2).

an elevated body temperature of $37.8^{\circ} \mathrm{C}$ and two masses in her right breast. The serum prolactin level was $108.0 \mathrm{ng} / \mathrm{mL}$, and mammary ultrasonography showed a right-sided external periareolar mass. Therefore, ultrasound-guided aspiration drainage was performed, and the pus was sent to our laboratory. Gram staining of the pus revealed several neutrophils and a few Gram-positive bacilli (Fig. 1). A small creamywhite colony of Corynebacterium spp. grew on 5\% sheep blood agar with capneic incubation and thioglycolate medium with enrichment approximately 72 hours after inoculation. The colony grew at an increased rate when the agar was supplemented with $1 \%$ Tween 80 . Initial routine biochemical identification of the corynebacterial strain using the API Coryne System (bioMérieux, Marcy-l'Etoile, France) identified C. argentoratense (profile No. 2040104) as the pathogen, with a probability of $87.1 \%$, whereas subsequent $16 \mathrm{~S}$ rRNA gene sequencing revealed the causative bacteria to be $C$. kroppenstedtii (sequence similarity to the C. kroppenstedtii DSM 44385 strain of 100\%). The isolate was sensitive to gentamycin, vancomycin, and linezolid (Table). Based on the findings of a previous study (2), doxycycline (100 mg BID) was administered, although it was discontinued shortly thereafter due to the development of gastrointestinal side effects. Levofloxacin was then administered
Table. Minimum Inhibitory Concentration of Various Antibiotics on Corynebacterium kroppenstedtii Isolated from Case1 and 2. Interpretation Results Were Based on the Criteria of Clinical and Laboratory Standards Institute (M45-A).

\begin{tabular}{ccccc}
\hline & \multicolumn{2}{c}{ Case 1 } & \multicolumn{2}{c}{ Case2 } \\
\hline & $\mathrm{MIC}(\mathrm{mg} / \mathrm{L})$ & $\begin{array}{c}\text { interpretation } \\
\text { result }\end{array}$ & $\mathrm{MIC}$ & $\begin{array}{c}\text { interpretation } \\
\text { result }\end{array}$ \\
\hline Ampicillin & $\geq 32$ & & 0.25 & \\
Imipenem/Cilastatin & $\geq 16$ & $\mathrm{R}$ & $\leq 0.25$ & $\mathrm{~S}$ \\
Gentamicin & 2 & $\mathrm{~S}$ & $\leq 0.25$ & $\mathrm{~S}$ \\
Erythromycin & $\geq 8$ & $\mathrm{R}$ & $\leq 0.12$ & $\mathrm{~S}$ \\
Clindamycin & $\geq 4$ & $\mathrm{R}$ & 0.12 & $\mathrm{~S}$ \\
Vancomycin & $\leq 0.5$ & $\mathrm{~S}$ & 1 & $\mathrm{~S}$ \\
Linezolid & $\leq 0.25$ & $\mathrm{~S}$ & $\leq 0.25$ & $\mathrm{~S}$ \\
\hline
\end{tabular}

MIC: minimum inhibitory concentration

S: susceptible

$\mathrm{R}$ : resistant

for eight weeks as a second-line drug, and the patient's symptoms gradually resolved. The drain was removed, and no relapse occurred during an observation period of two years. After withdrawing the dose of sulpiride, the serum prolactin level normalized.

\section{Case 2}

A 34-year-old nulliparous woman presented to our hospital with redness, pain and swelling of the right breast in December 2013. She had begun to experience an uncomfortable sensation in her right breast two weeks before the consultation. She had a history of schizophrenia and was currently taking fluphenazine, zolpidem and duloxetine. She was not allergic to any medications and did not smoke, although she was a social drinker. She was nulliparous and not pregnant. The findings of a physical examination revealed redness and swelling of the right breast (Fig. 2), with a serum prolactin level of $40.5 \mathrm{ng} / \mathrm{mL}$. Mammary ultrasonography showed a right-sided external periareolar dendritically expanded mass. Incision and drainage were performed, and the pus was sent to our laboratory. Gram staining of the pus revealed several neutrophils, but no bacteria. However, Corynebacterium spp. grew on 5\% sheep blood agar with capneic incubation 72 hours after inoculation. The colony grew at an increased rate when the agar was supplemented with $1 \%$ Tween 80 . Routine biochemical identification of the corynebacterial strain using the API Coryne System identified the pathogen as $C$. argentoratense (profile No. 2040104), with a probability of $87.1 \%$, whereas $16 \mathrm{~S}$ rRNA gene sequencing revealed the causative bacteria to be C. kroppenstedtii (sequence similarity to the C. kroppenstedtii DSM 44385 strain of 99.71\%). The isolate was sensitive to ampicillin, imipenem/cilastatin, gentamycin, erythromycin, clindamycin, vancomycin and linezolid (Table). Doxycycline (100 mg BID) was administered for eight weeks, and the discharge of pus gradually decreased. The incision was closed, and no relapse occurred during an observation period of 11 months. After withdrawing the dose of fluphenazine, the serum prolactin level normalized. 


\section{Discussion}

We herein reported two cases of granulomatous mastitis caused by $C$. kroppenstedtii infection in nulliparous young women. In both cases, the patients were initially treated for granulomatous mastitis. Granulomatous mastitis, an inflammatory disease of unknown etiology, is an uncommon benign disorder of the breast (1). It is most commonly seen in parous young women, often within a few years of pregnancy. Although systemic glucocorticoid therapy is often used successfully to treat symptomatic patients, partial or total mastectomy has been performed in some refractory cases, as granulomatous mastitis is suspected to be an autoimmune disease (3).

C. kroppenstedtii was first isolated from human sputum in 1998 (4). Bernard et al. reported 72 strains of rare or recently described Corynebacterium species (5). In their study, four of 72 strains were identified as C. kroppenstedtii, and one C. kroppenstedtii strain was isolated from a breast abscess.

In 2002, Paviour et al. reported 24 patients with mastitis (2), among whom Corynebacterium spp. were isolated from breast tissue, pus or deep wound swabs, with C. kroppenstedtii infection being the most common finding. Unlike many other Corynebacterium spp., C. kroppenstedtii contains tuberculostearic instead of mycolic acid in the cell wall and requires lipids for its growth (4). As seen in our two cases, supplementation with fatty acids increases the growth rate of C. kroppenstedtii. This lipophilic characteristic explains why $C$. kroppenstedtii is often found in the lipid-rich mammary glands (6). We suggest that the incubation time be extended if infection with C. kroppenstedtii is suspected, as the rate of colony growth is slower without fatty acid supplementation.

In order to identify which Corynebacterium spp. was responsible for the infections in our two cases, we first used the API Coryne System; this test identified $C$. argentoratense (profile No. 2100104) as the pathogen in both cases. Sugahara et al. also reported a case in which $C$. argentoratense was identified as the pathogen using API Coryne (7). Based on these findings, we suggest that the identification of $C$. argentoratense (profile No. 2100104) in a breast sample using API Coryne should raise suspicion regarding an infectious cause of C. kroppenstedtii and prompt further tests, such as 16S rRNA gene sequencing.

C. kroppenstedtii grows well in a lipid-rich environment. In previous reports, most cases of granulomatous mastitis caused by $C$. kroppenstedtii infection were observed in parous women, most likely because these women had developed mammary glands. Taylor et al. reported 34 cases of granulomatous mastitis due to Corynebacterium spp. (mainly C. kroppenstedtii) (6). Only two cases of nulliparous women with the same diagnosis have been reported in the literature. Both were on long-term phenothiazine regimens, which are known to increase the serum prolactin concentration. One woman also had hyperprolactinemia and galactorrhea. Additionally, cases of idiopathic granulomatous mastitis associated with pituitary prolactinoma or risperidone-induced hyperprolactinemia have been reported $(8,9)$. Both cases reported in this study also were associated with medicationinduced hyperprolactinemia (sulpiride and fluphenazine, respectively). Both of these drugs are known to increase the serum prolactin concentration $(10,11)$. The lactational changes induced by drug-induced hyperprolactinemia may increase the risk of developing granulomatous mastitis after C. kroppenstedtii infection.

Treating granulomatous mastitis is difficult and controversial. Drainage for the pus is the most important treatment strategy, whereas antibiotic treatment is often ineffective despite the pathogen having been shown to be susceptible to antibiotics in vitro (2). Furthermore, an impaired neutrophil response to the Nod2 agonist associated with corynebacterial infection was recently reported to be partially responsible for this phenomenon (12). Paviour et al. suggested using doxycycline due its greater lipid solubility. In the current two cases, treatment was started with doxycycline, which was effective for one patient. In the other case, the medication was changed to levofloxacin as a result of the patient's doxycycline intolerance. Further studies are needed to assess appropriate treatment strategies for granulomatous mastitis.

\section{The authors state that they have no Conflict of Interest (COI).}

\section{Financial Support}

This work was supported by a grant from the National Center for Global Health and Medicine (25-114).

\section{References}

1. Al-Khaffaf B, Knox F, Bundred NJ. Idiopathic granulomatous mastitis: A 25-year experience. J Am Coll Surg 206: 269-273, 2008.

2. Paviour S, Musaad S, Roberts S, et al. Corynebacterium species isolated from patients with mastitis. Clin Infect Dis 35: 14341440, 2002.

3. Wilson JP, Massoll N, Marshall J, Foss RM, Copeland EM, Grobmyer SR. Idiopathic granulomatous mastitis: in search of a therapeutic paradigm. Am Surg 73: 798-802, 2007.

4. Collins MD, Falsen E, Akervall E, Sjöden B, Alvarez A. Corynebacterium kroppenstedtii sp. nov., a novel corynebacterium that does not contain mycolic acids. Int J Syst Bacteriol 48: 14491454, 1998.

5. Bernard KA, Munro C, Wiebe D, Ongsansoy E. Characteristics of rare or recently described corynebacterium species recovered from human clinical material in Canada. J Clin Microbiol 40: 43754381, 2002.

6. Taylor GB, Paviour SD, Musaad S, Jones WO, Holland DJ. A clinicopathological review of 34 cases of inflammatory breast disease showing an association between corynebacteria infection and granulomatous mastitis. Pathology 35: 109-119, 2003.

7. Sugahara Y, Ohkusu K, Ohtsuka Y, et al. A case of recurrent mastitis caused by Corynebacterium kroppenstedtii. Nihon Rinsho Biseibutsugaku Zasshi (J Clin Microbiol) 22: 161-166, 2012 (in Japanese, Abstract in English).

8. Rowe PH. Granulomatous mastitis associated with a pituitary prolactinoma. Br J Clin Pract 38: 32-34, 1984. 
9. Lin $\mathrm{CH}$, Hsu CW, Tsao TY, Chou J. Idiopathic granulomatous mastitis associated with risperidone-induced hyperprolactinemia. Diagn Pathol 7: 2, 2012.

10. Mancini AM, Guitelman A, Vargas CA, Debeljuk L, Aparicio NJ. Effect of sulpiride on serum prolactin levels in humans. J Clin Endocrinol Metab 42: 181-184, 1976.
11. Chalmers RJ, Bennie EH. The effect of fluphenazine on basal prolactin concentrations. Psychol Med 8: 483-486, 1978.

12. Bercot B, Kannengiesser C, Oudin C, et al. First description of NOD2 variant associated with defective neutrophil responses in a woman with granulomatous mastitis related to corynebacteria. J Clin Microbiol 47: 3034-3037, 2009.

(C) 2015 The Japanese Society of Internal Medicine http://www.naika.or.jp/imonline/index.html 